\title{
Inactivation of an integrated antibiotic resistance gene in mammalian cells to re-enable antibiotic selection
}

\author{
Peiling Ni, Qian Zhang, Haixia Chen, and Lingyi Chen \\ State Key Laboratory of Medicinal Chemical Biology, 2011 Collaborative \\ Innovation Center of Tianjin for Medical Epigenetics, and College of Life \\ Sciences, Nankai University. Tianjin, China
}

BioTechniques 56:198-201 (April 2014) doi 10.2144/000114160

Keywords: antibiotic resistance gene; genetic engineering; CRISPR/Cas

Supplementary material for this article is available at www.BioTechniques.com/article/114160.

Removing an antibiotic resistance gene allows the same antibiotic to be re-used in the next round of genetic manipulation. Here we applied the CRISPR/Cas system to disrupt the puromycin resistance gene in an engineered mouse embryonic stem cell line and then reused puromycin selection in the resulting cells to establish stable reporter cell lines. With the CRISPR/Cas system, pre-engineered sequences, such as loxP or FRT, are not required. Thus, this technique can be used to disrupt antibiotic resistance genes that cannot be removed by the Cre-loxP and Flp-FRT systems.

Antibiotic resistance genes are widely used in genetic engineering, including homologous recombination and transgenic studies. However, there are only limited number of antibiotic resistance genes available for use in mammalian cells, including the puromycin (puro), neomycin (neo), and hygromycin (bygro) resistance genes. For the multi-step genetic modifications of the same mammalian cell line, each step requires selection using a distinct antibiotic resistance gene. Thus, the number of modifications is restricted by the number of available antibiotic resistance genes.

To circumvent this restriction, the integrated antibiotic resistance genes could be removed by the Cre-loxP or Flp-FRT systems $(1,2)$. However, flanking lox $\mathrm{P}$ or FRT sites must be added upstream and downstream of the antibiotic resistance gene before the recombination vector is integrated into a mammalian genome. In some cases, genetic modifications have been engineered in mammalian cells, and exogenous antibiotic resistance genes are not flanked by loxP or FRT sites. Thus, it is impossible to remove these antibiotic resistance genes by Cre or Flp recombinase.

For example, the $\mathrm{KH} 2$ mouse embryonic stem (ES) cell line is engineered for a doxycycline-inducible expression system. It harbors a constitutively expressed M2rtTA transactivator from the Rosa 26 endogenous promoter and a modified locus downstream of ColA1 for exogenous gene insertion through the Flp-in system. After these genetic modifications, KH2 ES cells have puro and neo genes, as well as a bygro gene lacking a promoter and an ATG initiation codon, which is to be complemented by the Flp-in plasmid. The neo gene with flanking FRT sites is removed by Flp-mediated recombination upon Flp expression. Thus, once the exogenous gene is introduced into KH2 ES cells to make an doxycyclineinducible cell line, the cells are resistant to puromycin and hygromycin (3). The multiple resistance genes make it inconvenient to perform additional genetic engineering in such cell lines. Similarly, the doxycycline-inducible ES cell line AinV 15 has the same issue (4).

To allow further genetic manipulation of $\mathrm{KH} 2$ cells, we applied the CRISPR/Cas system to disrupt its puro gene $(5,6)$. We used the CRISPR Design Tool (http://crispr.mit.edu/) to search for potential guide sequences in the first $250 \mathrm{bp}$ of the puro gene (7) and 13 sequences were identified. Two additional criteria were applied to select the guide sequence: (i) genomewide off-target sites of binding to the guide sequence have to contain at least four mismatches to minimize off target effects, and (ii) the target site should be close to the $5^{\prime}$ end of the puro gene to ensure that a large portion of the gene will be affected by a Cas9-caused frameshift mutation. With these two criteria, a guide sequence (CACGCGCCACACCGTCGATC) located +96 to +115 bp of the puro gene was chosen (Figure 1A). Sequence analysis revealed that all potential off-target binding sites in both the human and mouse genomes have at least four mismatches to this guide sequence (Supplementary Table S1). In addition, at least $1 \mathrm{mismatch}$ in each off-target binding site is within the 8 to 12 base long seed sequence at the $3^{\prime}$ end of the guide sequence, which is critical for the DNA cleavage specificity. It has been reported that Cas9 cleavage is dramatically reduced at DNA sites with three or more interspaced mismatches (7-9). Thus, the chosen guide sequence should minimize the off-target effects of the CRISPR/Cas system.

To construct the plasmid expressing Cas 9 and the single-guide RNA (sgRNA), a pair of oligos (5'-CACCGCACGCGCCACACCGTCGATC, and 5'-AAACGATCGACGGTGTGGCGCGTGC) containing the guide

Method summary:

This method uses the CRISPR/Cas system to disrupt antibiotic resistance genes in mammalian cells, thus facilitating additional genetic manipulation with the same antibiotic selection. 


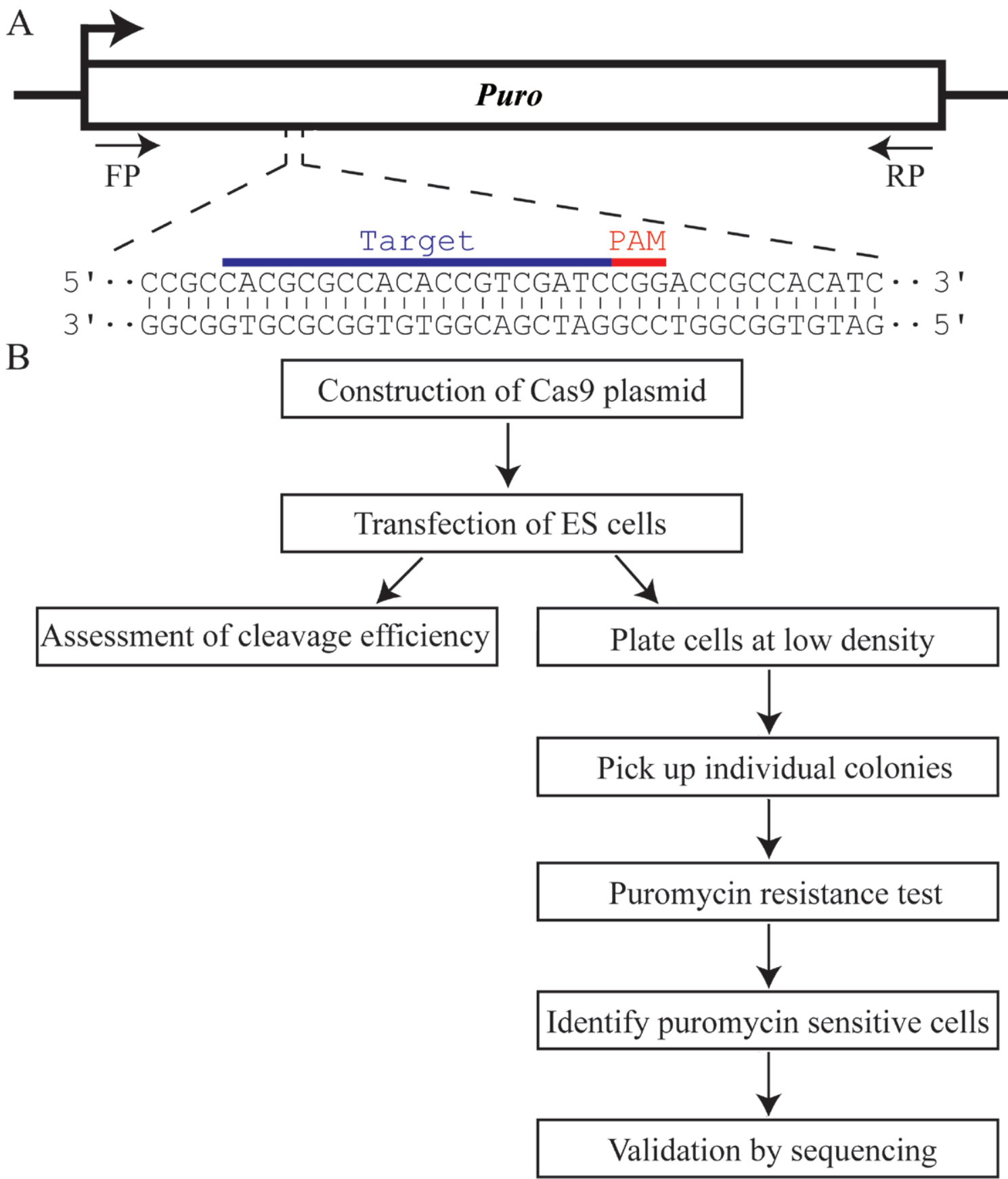

Figure 1. Guide sequence design and experimental outline. (A) A 20 bp sequence (highlighted with a blue line) located in the coding region of the puro gene (shown as an open rectangle) is selected as the guide sequence. The PAM sequence (CGG) lies immediately to the $3^{\prime}$ end of the guide sequence and is marked with a red line. Arrows represent the forward and reverse primers (labeled as FP and RP) for PCR amplification of the puro gene. (B) A flowchart of the experimental procedure.

sequence were annealed and ligated to the BbsI-linearized vector pX330 (6). The resulting plasmid was transfected into $\mathrm{KH} 2$ cells using Lipofectamine 2000 (Invitrogen, Carlsbad, CA) following the manufacturer's instruction. For 1 well of a 6 -well plate, $4 \mu \mathrm{g}$ Cas 9 plasmid was transfected into $5 \times 10^{5}$ cells. The cells were harvested 48 hours after transfection.
A small fraction of the cells was plated at low density to form singlecell clones, while the rest were used to estimate the cleavage efficiency at the puro gene using the T7 Endonuclease I assay. In brief, the genomic DNA was purified from KH2 cells, and the puro gene was PCR amplified with forward (FP: GAGTACAAGCCCACGGT) and reverse (RP: GAGCCGCTC-
GTAGAAGG) primers. The PCR products were purified using the EasyPure PCR Purification Kit (TransGen, Beijing, China). Then, $500 \mathrm{ng}$ PCR product was mixed with $2 \mu \mathrm{l} 10 \times$ NEBuffer 2 (New England Biolabs, Ipswich, MA) and $\mathrm{H}_{2} \mathrm{O}$ to a final volume of $19 \mu \mathrm{l}$. The mix was incubated at $95^{\circ} \mathrm{C}$ for $15 \mathrm{~min}, 65^{\circ} \mathrm{C}$ for $30 \mathrm{~min}$, room temperature for $30 \mathrm{~min}$, 
A

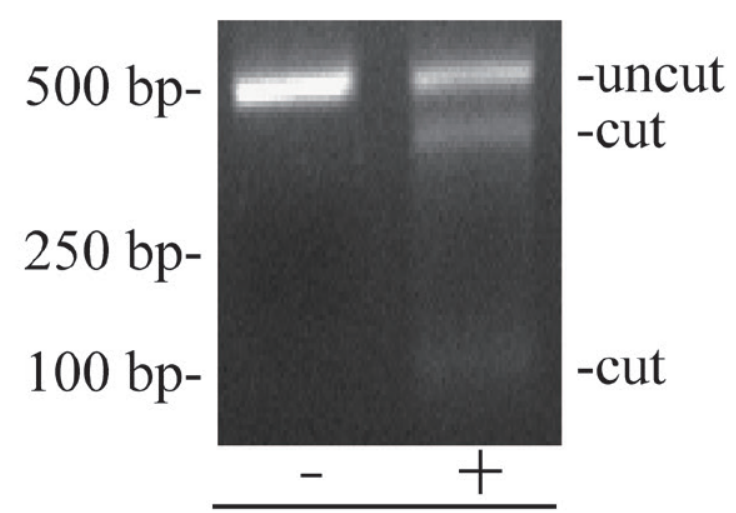

T7 Endonuclease I

C

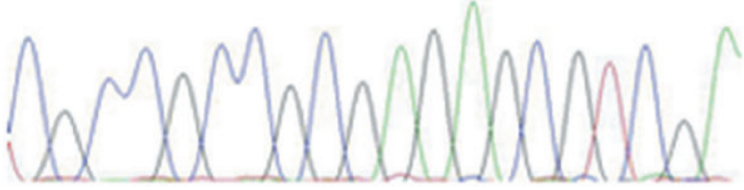

CGCCGCCGCGAGAGCGTCGA
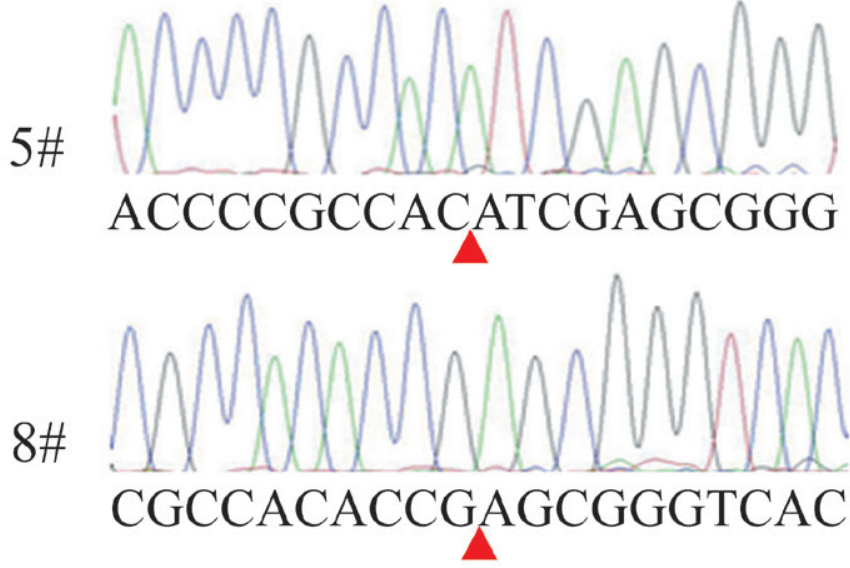

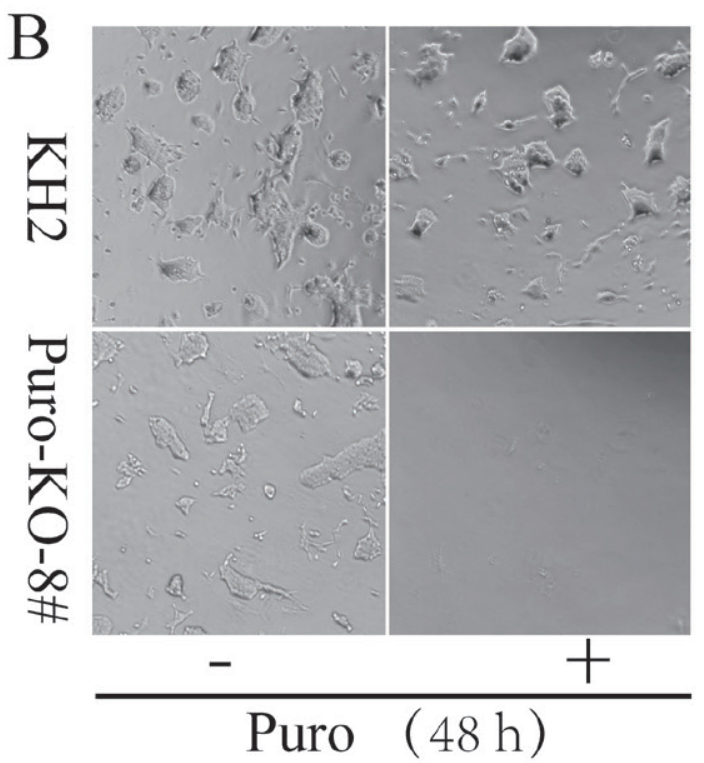

D
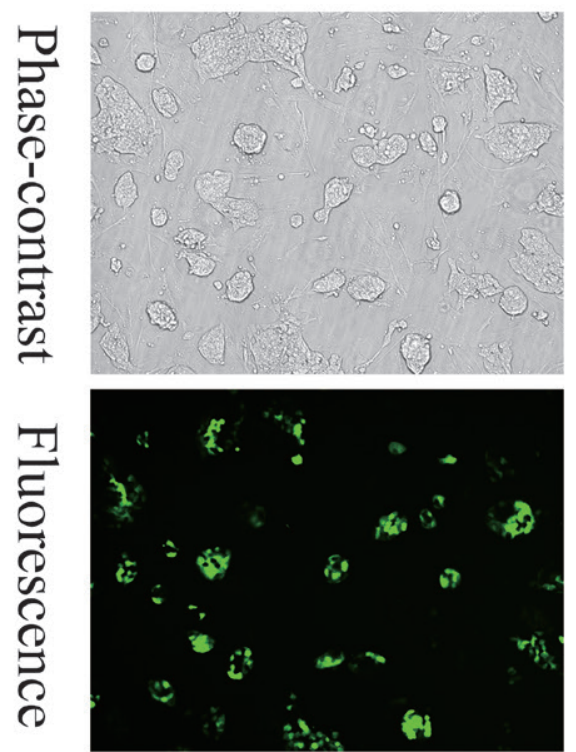

WT

Target

PAM

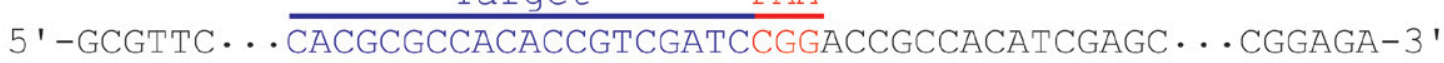

$4 \#(\Delta 160)$

5 '-GCG-

$-A G A-3^{\prime}$

$5 \#(\triangle 28)$

5 '-GCGTTC $\cdots$. CAC

-ATCGAGC $\cdots$ CGGAGA-3'

8\#( $\triangle 21) 5^{\prime}-$ GCGTTC $\cdots$ CACGCGCCACACCG

$-A G C \cdots$ CGGAGA-3'

Figure 2. Disruption of the puro gene in KH2 cells and establishment of a pNanog-GFP reporter cell line in Puro-K0-8\# cells. (A) T7 endonuclease I assay to assess the cleavage efficiency at the puro gene. (B) Puromycin resistance test on the cell lines after Cas9 cleavage. Cells expanded from individual colonies were cultured in medium with or without $2.5 \mu \mathrm{M}$ puromycin for $48 \mathrm{~h}$. Phase contrast images are shown. (C) Sequencing result of the puro gene in three puromycin sensitive cell lines (Puro-KO-4\#, Puro-KO-5\#, and Puro-KO-8\#). Red triangles in the sequencing chromatographs mark the deletion sites in the three cell lines. (D) Phase contrast and fluorescence images of a pNanog-GFP reporter cell line established from Puro-KO-8\# using puromycin selection. Not all cells are GFP positive due to the heterogeneity of the Nanog promoter activity in mouse ES cells (10). 
and on ice for $30 \mathrm{~min}$ to form heteroduplex DNA. Following the incubation, $1 \mu \mathrm{l}$ of T7 endonuclease I (New England Biolabs) was added to the DNA, and the reaction mix was incubated at $37^{\circ} \mathrm{C}$ for $1 \mathrm{~h}$. The resulting DNA sample was separated by agarose gel electrophoresis to determine the size of DNA fragments. As shown in Figure 2A, the cleavage efficiency at the puro gene is approximately $30 \%$.

After five days in culture, colonies had formed from single cells. Forty individual colonies were picked up and expanded. To test whether the puro gene is disrupted in these clones, cells were grown in medium supplemented with puromycin. After 48 h of puromycin selection, puromycin-sensitive cells all died out, while puromycin-resistant cells continued growing (Figure 2B). Out of 40 picked colonies, 8 cell lines were identified as containing puromycin-sensitive cells. The rate of puro disruption is close to the cleavage efficiency at the puro gene.

The puro gene was further analyzed in three cell lines (Puro-KO-4\#, Puro-KO-5\#, and Puro-KO-8\#) by PCR and sequencing. These 3 clones had 160, 28, and 21 bp deletions in the puro gene, respectively (Figure $2 \mathrm{C}$ ). The 160 and 28 bp deletion in Puro-KO-4\# and Puro-KO-5\# caused frameshifts, whereas the $21 \mathrm{bp}$ deletion in Puro-KO-8\# was an in-frame mutation, leading to a 7 amino acid residue deletion in the protein. To knock out a gene with CRISPR/Cas, frameshift mutations are preferred. However, we screened puro gene disruption with a functional puromycin resistance test. Therefore, both frameshift and in-frame mutations are acceptable, as long as the cells become sensitive to puromycin.

To demonstrate that disruption of the puro gene allows re-selection by puromycin, we re-introduced a plasmid carrying the GFP-IRES-puro gene driven by the Nanog promoter (pNanogGFP) into the Puro-KO-8\# cells. After 10 days of puromycin selection, stable cell lines expressing GFP could be established (Figure 2D).

So far, we have demonstrated that CRISPR/Cas efficiently disrupts the puro gene in mammalian cells, allowing puromycin selection in the resulting cell line for additional genetic manipulation. Given the simple design of the CRISPR/Cas system, it is easy to extend the application to other antibiotic resistance genes, facilitating multiple genetic engineering steps. Our strategy for selecting the guide sequence for the puro gene is also applicable to other antibiotic resistance genes. In comparison with the Cre-loxP or Flp-FRT system, the CRISPR/Cas system does not require pre-existing loxP or FRT sites, allowing disruption of an antibiotic resistance gene lacking flanking loxP or FRT sites. In addition, CRISPR/Cas can be multiplexed to target several antibiotic resistance genes simultaneously, whereas Cre-lox $\mathrm{P}$ is less useful for targeting multiple genes because multiple lox $P$ sites might lead to undesirable recombination and genome instability.

\section{Author contributions}

L.C. designed the experiment. P.N., Q.Z., and H.C. performed research. L.C. and P.N. wrote and edited the paper.

\section{Acknowledgments}

This work is supported by the National Natural Science Foundation of China (Grant No. 31271547), the National Key Basic Research and Development Program of China (Grant No. 2010CB833603), the Program for New Century Excellent Talents (NCET-13-0293), the 111 Project Grant (B08011), and the Funds for National Basic Science Personnel Training (No. J1103503).

\section{Competing interests}

The authors declare no competing interests.

\section{References}

1. Sauer, B. and N. Henderson. 1988. Sitespecific DNA recombination in mammalian cells by the Cre recombinase of bacteriophage P1. Proc. Natl. Acad. Sci. USA 85:5166-5170.

2. Sadowski, P.D. 1995. The Flp recombinase of the 2-microns plasmid of Saccharomyces cerevisiae. Prog. Nucleic Acid Res. Mol. Biol. 51:53-91.

3. Beard, C., K. Hochedlinger, K. Plath, A. Wutz, and R. Jaenisch. 2006. Efficient method to generate single-copy transgenic mice by site-specific integration in embryonic stem cells. Genesis 44:23-28.

4. Kyba, M., R.C. Perlingeiro, and G.Q. Daley. 2002. HoxB4 confers definitive lymphoid-myeloid engraftment potential on embryonic stem cell and yolk sac hematopoietic progenitors. Cell 109:29-37.

5. Cong, L., F.A. Ran, D. Cox, S. Lin, R. Barretto, N. Habib, P.D. Hsu, X. Wu, et al. 2013. Multiplex genome engineering using CRISPR/Cas systems. Science 339:819-823.
6. Mali, P., L. Yang, K.M. Esvelt, J. Aach, M. Guell, J.E. DiCarlo, J.E. Norville, and G.M. Church. 2013. RNA-guided human genome engineering via Cas9. Science 339:823-826.

7. Hsu, P.D., D.A. Scott, J.A. Weinstein, F.A. Ran, S. Konermann, V. Agarwala, Y. Li, E.J. Fine, et al. 2013. DNA targeting specificity of RNA-guided Cas9 nucleases. Nat. Biotechnol. 31:827-832.

8. Mali, P., J. Aach, P.B. Stranges, K.M. Esvelt, M. Moosburner, S. Kosuri, L. Yang, and G.M. Church. 2013. CAS9 transcriptional activators for target specificity screening and paired nickases for cooperative genome engineering. Nat. Biotechnol. 31:833-838.

9. Yang, H., H. Wang, C.S. Shivalila, A.W. Cheng, L. Shi, and R. Jaenisch. 2013. One-step generation of mice carrying reporter and conditional alleles by CRISPR/Cas-mediated genome engineering. Cell 154:1370-1379.

10. Singh, A.M., T. Hamazaki, K.E. Hankowski, and N. Terada. 2007. A heterogeneous expression pattern for Nanog in embryonic stem cells. Stem Cells 25:2534-2542.

Received 09 January 2014; accepted 06 February 2014.

Address correspondence to Lingyi Chen, College of Life Sciences, Nankai University, Tianjin, China.E-mail: lingyichen@nankai.edu.cn

To purchase reprints of this article, contact: biotechniques@fosterprinting.com 